\title{
Modeling domain wall dynamics in thin magnetic strips with disorder
}

\author{
Lasse Laurson $^{1}$, Adil Mughal ${ }^{1}$, Gianfranco Durin ${ }^{1,2}$, and Stefano Zapperi ${ }^{1,3}$ \\ ${ }^{1}$ ISI Foundation, Viale S. Severo 65, 10133 Torino, Italy. \\ ${ }^{2}$ INRIM, Strada delle Cacce 91, 10135 Torino, Italy. \\ ${ }^{3}$ CNR-INFM, S3, Università di Modena e Reggio Emilia, Via Campi 213/A, 41100 Modena, Italy.
}

\begin{abstract}
We present a line-based model of transverse domain walls in thin magnetic strips, to study the effect of bulk disorder on the domain wall dynamics within the thermally activated creep regime. The creep velocity is found to exhibit a non-linear dependence on both applied magnetic fields and electric currents, characterized by similar creep exponents for both forms of the external drive. We discuss briefly the significance of the inherently stochastic thermally activated domain wall motion from the point of view of spintronics applications, where it generally is essential to be able to control the domain wall displacement in a deterministic manner.
\end{abstract}

\section{INTRODUCTION}

D OMAIN wall dynamics in thin magnetic strips or wires driven by either applied external magnetic fields or electric currents has been an active field of research during recent years, due to both the fundamental aspects of the underlying physics as well as due to the potential spintronics applications [1], [2], [3], [4]. Most of the theoretical studies of such phenomena have so far focused on "perfect" systems free of any imperfections in the sample that could affect the dynamics of the domain wall. However, disorder is present in practically any realistic material, either in the form of edge roughness or various point-like defects in the bulk of the system. While the effect of edge roughness [5], [6], [7] or notches [8] on domain wall motion has received much attention in the literature, bulk disorder is usually assumed to be insignificant in narrow nanostrips. However, this might not be true in general: for instance, thickness fluctuations of the strip might give rise to bulk disorder similarly to the disorder due to the rough edges of the system. Also other forms of impurities might be present in the bulk of the system, affecting the domain wall dynamics.

In this paper we present a line-based model of a transverse domain wall in a narrow and thin disordered magnetic (nano)strip. Such a coarse grained model is useful in understanding the effect of various kinds of disorder on the domain wall dynamics, because it does not suffer from the shortcomings of some other approaches such as micromagnetic simulations where inclusion of disorder is tricky due to meshrelated problems, or point-particle models in which the internal degrees of freedom of the domain wall are not considered. We focus on the effect of randomly distributed point-like pinning centers in the bulk of the system on the domain wall dynamics in the sub-threshold thermally activated creep regime. In some recent studies, it has been demonstrated that the experimentally relevant domain wall velocities often are within this regime [5]. The paper is organized as follows: in the next Section, the line based model of a transverse domain wall is presented, and some numerical results on the creep motion of such a domain wall are presented in Section III. Section IV finishes the paper with discussion and conclusions.

\section{MODEL}

We consider here strips of length $L$, width $W$ and thickness $D$, satisfying $L \gg W \gg D$, made of a soft magnetic material such as Permalloy. The domain wall structure in such a strip depends on the balance between exchange and anisotropy energies. The latter is here taken to be dominated by shape anisotropy, and thus the domains lie along the long axis of the strip. We focus on the case in which the width $W$ and thickness $D$ of the strip are sufficiently small, so that the stable domain wall structure is the so called transverse wall [9], [10], [11]. For an example of its micromagnetic structure, see the top panel of Fig. 1. For wider and/or thicker strips (not considered here), a domain wall with vortex topology would have a lower energy.

Due to the balance between exchange interactions and the effect of the demagnetizing fields $\vec{H}_{d m}$ arising from the "magnetic charges" within the transverse domain wall, the equilibrium shape of the wall resembles the letter V. These charges are associated to the discontinuities of the normal component of the magnetization. A simplified description of the wall structure is obtained by considering the wall to be composed of two $90^{\circ}$ domain walls, with the first one from the left in Fig. 1 separating domains with spins pointing to the right and up, respectively, while the second $90^{\circ}$ wall separates the domain with spins up from from the one with spins to the left. Clearly, such a domain description cannot be completely accurate as the magnetization within the wall rotates smoothly from right to left as in Fig. 1, so the magnetic charges associated to this rotation are actually distributed across the transition region. Nevertheless, by imposing the above approximation of two 90-degree walls with the magnetic charges concentrated along these walls yields a qualitatively correct equilibrium shape for the transverse wall, i.e. the two 90-degree walls form a V-shaped structure. 
The lower panel of Fig. 1 shows a schematic of the model. The two lines, labeled (A) and (B) in the lower panel of Fig 11 have line tensions $\gamma_{w}$, and interact repulsively due to the exchange interaction. With the local width of the wall $w(y)$, exchange interactions along the strip axis lead to an energy $E_{e} \sim 1 / w(y)$, resulting in a repulsive force $F_{e}(y) \sim 1 / w(y)^{2}$ between any pair of line segments with the same $y$-coordinates. These forces are balanced by the forces due to the demagnetizing fields arising from the magnetic charges. The top edge (with $y=W$ ) has a positive charge density $\sigma=M_{s}$, where $M_{s}$ is the saturation magnetization. Similarly, the bottom edge (where $y=0$ ) has $\sigma=-M_{s}$. As described above, also the lines are charged: whenever the local angle between the line and the $x$-axis is different from 45 degrees, the lines have a non-zero charge density. Notice that the total charge of the domain wall is counterbalanced by the negative charges at the two ends of the strip (with $x=0$ and $x=L$ ).

Furthermore, the domain wall can be driven towards the positive $x$ direction either by applying an external magnetic field $\vec{H}_{\text {ext }}=H_{\text {ext }} \hat{x}$ or an electric current $\vec{j}=-\jmath \hat{x}$ (with the direction chosen in such a way that the electrons move along the $+\hat{x}$ direction). Here we restrict ourselves to the case of low fields and currents: For stronger driving one should also include a "phase" angle of the domain wall magnetization as a variable to the model. For the moment we assume that all magnetic moments remain within the plane of the strip. In other words, the fields and currents are restricted to be well below the so called Walker threshold, where precession of the domain wall magnetization about the long axis of the strip would take place (something that in the strip geometry considered here would in fact proceed via nucleation and propagation of an antivortex). To see how the effect of a small current can be included in the present model, we point out that the Landau-Lifshitz-Gilbert (LLG) equation describing the time evolution of the magnetization $\vec{M}(\vec{r}, t)$, with the additional terms describing the effect of current can be written [12]

$$
\begin{aligned}
\frac{\partial \vec{M}}{\partial t}= & -\gamma \vec{M} \times \vec{H}_{e f f}+\alpha \vec{M} \times \frac{\partial \vec{M}}{\partial t}-v_{j} \frac{\partial \vec{M}}{\partial x}+ \\
& +\beta v_{j} \vec{M} \times \frac{\partial \vec{M}}{\partial x},
\end{aligned}
$$

where $\vec{H}_{\text {eff }}$ is the total effective magnetic field, $\gamma$ is the gyromagnetic ratio and the second term is the Gilbert damping term. The effects due to an electric current are taken into account by the last two terms, usually referred to as the adiabatic and non-adiabatic terms, respectively. The essential point here is that the effect of current enters in both terms through the gradient of $\vec{M}$ : The non-adiabatic term enters like a magnetic field that is proportional to $\partial \vec{M} / \partial x$, while the adiabatic term exerts a torque on the spins of the domain wall, which is again proportional to $\partial \vec{M} / \partial x$. Thus, the simplest way to include the effects of the current is to add a local magnetic field which is proportional to the rate of change of the magnetization across the domain wall, i.e. to $1 / w(y)$. Thus, the difference between the two forms of driving is that whereas
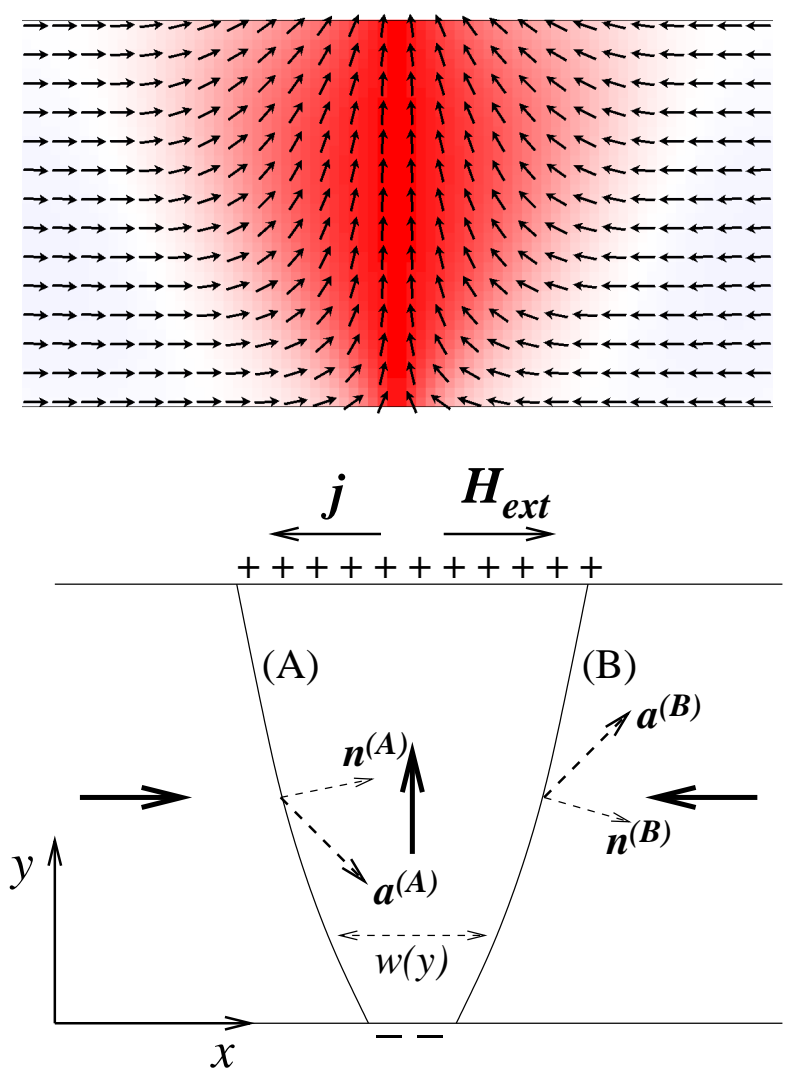

Fig. 1. Top: An example of a transverse domain wall from micromagnetic simulations (using the OOMMF program [13]), with the material parameters those of Permalloy, and strip dimensions $L=5 \cdot 10^{-6} \mathrm{~m}, W=160 \cdot 10^{-9} \mathrm{~m}$ and $D=8 \cdot 10^{-9} \mathrm{~m}$. Bottom: A schematic of the line-based model, where two lines (labeled (A) and (B), respectively) separating three different magnetic domains (with the magnetization direction indicated by the thick solid arrows) form a model of the transverse wall. The dashed arrows indicate the directions $\vec{a}^{(i)}(i=A, B)$ of the components of the local field which drive the lines to the positive $x$ direction, as well as the line normals $\vec{n}^{(i)}$.

the effect of the field is independent of the local domain wall width, the same is not true for the current drive.

The total magnetic field due to all these contributions is then evaluated at each segment of the discretized lines. While the domain wall as a whole is driven towards the $+\hat{x}$ direction by a field along the positive $x$ direction, the same is not true for the two "sub-walls" separately: Due to the different spin orientations on each side of the two sub-walls, the relevant field components driving the sub-walls are also different from each other. For the line (A), the relevant component of the local magnetic field driving the line towards the $+\hat{x}$ direction is the component along $\vec{a}^{(A)}=1 / \sqrt{2}(\hat{x}-\hat{y})$, while the relevant component for line (B) is along $\vec{a}^{(B)}=1 / \sqrt{2}(\hat{x}+\hat{y})$. Notice that the total field acting on the domain wall as a whole is along the positive $x$ direction $\left(\vec{a}^{(A)}+\vec{a}^{(B)}=\sqrt{2} \hat{x}\right)$. A force with a magnitude of these components of the local magnetic field is then taken to act on each segment of the lines along the local normal $\vec{n}_{j}$. This leads to a local normal force along each segment of the two lines, which is then used to calculate the dynamics of the system. To account for the effect of the finite damping coefficient in Eq. (3), we introduce a domain wall mass $m$, along with a friction force, so that the equation 
of motion for the line segment $j$ reads

$$
\begin{aligned}
m \frac{d \vec{v}_{j}}{d t}= & -\chi \vec{v}_{j}+\left[-\gamma_{w} \kappa_{j}+F_{e, j}^{(i)}-\left(\nabla U_{p}\right) \cdot \vec{n}_{j}+\right. \\
& \left.+\left(\vec{H}_{d m, j}+\vec{H}_{e x t, j}\right) \cdot \vec{a}^{(i)}+\eta_{j}\right] \vec{n}_{j}
\end{aligned}
$$

where $\chi$ is the effective friction coefficient, $\gamma_{w}$ is the line tension, $\kappa_{j}$ is the local curvature of the line and $\vec{n}_{j}$ is the local normal vector of the line. $F_{e, j}^{(A)}=-b /[w(y)]^{2}$ and $F_{e, j}^{(B)}=+b /[w(y)]^{2}$ are the repulsive forces between the two line segments of the different lines with the same $y$ coordinate due to exchange interactions. $U_{p}(\vec{r})=-C \sum_{j} e^{-\frac{1}{2}\left(\frac{\vec{r}-\vec{r}_{j}}{r_{0}}\right)^{2}}$ is a quenched random potential describing the interaction of the line segments with various defects localized at random positions $\vec{r}_{j}$ within the strip (with $r_{0}$ the range of interaction). $\vec{H}_{d m, j}$ and $\vec{H}_{e x t, j}$ are the demagnetizing and external fields acting on the line segment $j$, respectively. As discussed earlier, we include the effects due to an electric current as a local field with a magnitude inversely proportional to $w(y)$, by setting $\vec{H}_{e x t, j}=\left[H_{e x t}-\jmath / w(y)\right] \hat{x}$, where $H_{\text {ext }}$ is the magnitude of the applied external field and $\jmath$ is the applied current density. Finally thermal effects are included by a random force $\eta_{j}$ acting on each segment $j$ of the line, satifying $\left\langle\eta_{j}(t)\right\rangle=0$ and $\left\langle\eta_{j}(t) \eta_{k}\left(t^{\prime}\right)\right\rangle=k_{b} T \chi \delta_{j, k} \delta\left(t-t^{\prime}\right)$.

\section{NUMERICAL RESULTS}

The model is implemented by using the software package Surface Evolver [14], within which the line is taken to be formed by a set of vertices connected to each other by edges. The program allows automatic remeshing of the discretized line, with the line moving in continuous space, thus avoiding spurious lattice effects. The equations of motion are integrated numerically with the Euler algorithm. In the absense of disorder, thermal effects and external drive, the initial configuration of two parallel lines relax to reach a V-shaped stable structure, with the angle of the V-shape depending on the relative magnitude of the demagnetizing fields and effects arising from the exchange interactions (the line tension of the lines, the repulsive interaction between the two). After this initial relaxation, the external drive, the interaction with disorder and thermal effects are turned on and the evolution of the system is monitored. In the spirit of the model (where we imposed a restriction that the external driving is weak), we focus on the thermally activated subthreshold creep regime. The parameters of the simulations in dimensionless units of Eq. (2) are as follows: $\chi=1, \gamma_{w}=1, b=0.025, m=0.001$, $C=0.2$ and $r_{0}=0.01$. The demagnetizing field due to a charge $M_{s} \sigma d l$ (with $\sigma$ measuring the charge density in units of the saturation magnetization $M_{s}$ ) within a line segment of length $d l$ positioned at the origin is taken have a magnitude $M_{s} \sigma d l / r^{2}$, where the saturation magnetization is taken to have a value $M_{s}=0.15$, and $r$ is the distance from the origin. The unit of length is set by the sample dimensions, $L=4.5$ and $W=1.2$ (the very small thickness $D$ of the strip is neglected by treating the system as two-dimensional). Fig. 2 shows an example of a sequence of configurations of the creeping domain wall in the field driven case.
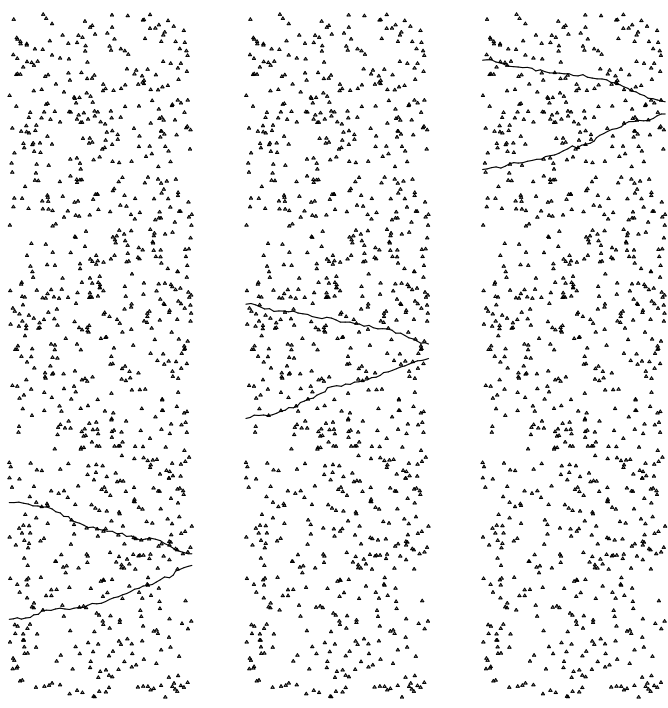

Fig. 2. Three snapshots for different times $t$ (from left to right, $t=5$, $t=17.5$ and $t=30$ in dimensionless units) of the domain wall moving in a disordered nanostrip (with the dots representing the pinning centers) under the influence of a subthreshold external field $H_{\text {ext }}$ and thermal fluctuations.

The dynamics of the domain wall in this regime displays the typical characteristics of creep motion: The domain wall moves in discrete jumps, separated by periods of pinning, see Fig. 3 for examples of the domain wall position as a function of time. Due to the thermally activated nature of the motion, the dynamics is inherently stochastic, and the relative fluctuations of the dynamics become increasingly pronounced as the driving force is deacreased.

The average creep velocity is found to exhibit non-linear dependence on both external field and current. In general, for creep motion of elastic manifolds subject to an applied force $f$ and temperature $T$, one expects the creep velocity to obey

$$
v(f, T) \sim e^{-\frac{E_{c}}{k_{b} T}\left(\frac{f_{c}}{f}\right)^{\mu}},
$$

where $\mu$ is the creep exponent, characterizing the divergence of the height of the energy barriers as the driving force tends towards zero. Our results presented in Fig. 4 indicate that for both external field and current (i.e. $f=H_{\text {ext }}$ and $f=\jmath$ ), the data is consistent with the above form with $\mu=0.4 \pm$ 0.1 . A more detailed study of these issues will be published elsewhere.

\section{Conclusions}

In this paper we have presented a line-based model of a transverse domain wall in a disordered magnetic nanostrip, and studied its dynamics within the thermally activated subthreshold creep regime. The creep motion of the domain wall interacting with point-like pinning centers randomly positioned within the bulk of the strip is found to exhibit typical features of creep of elastic manifolds, i.e. stochastic velocity fluctuations and a non-linear relation between the average creep velocity and the external drive. The finding that the creep exponents for the field and current driven cases are similar indicates a universality of the two forms of driving in the present case. This is in contrast with experiments on 

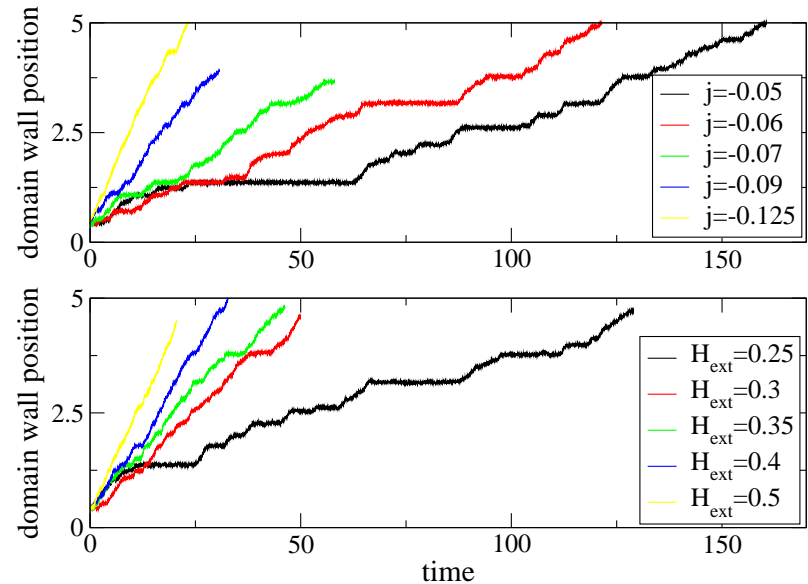

Fig. 3. Examples of the domain wall position along the long axis of the strip as a function of time for different magnitudes of the external drive within the creep regime. Top panel shows the current drive, while field drive is presented in the lower panel. Notice that the dynamics becomes increasingly erratic as the driving force is decreased.
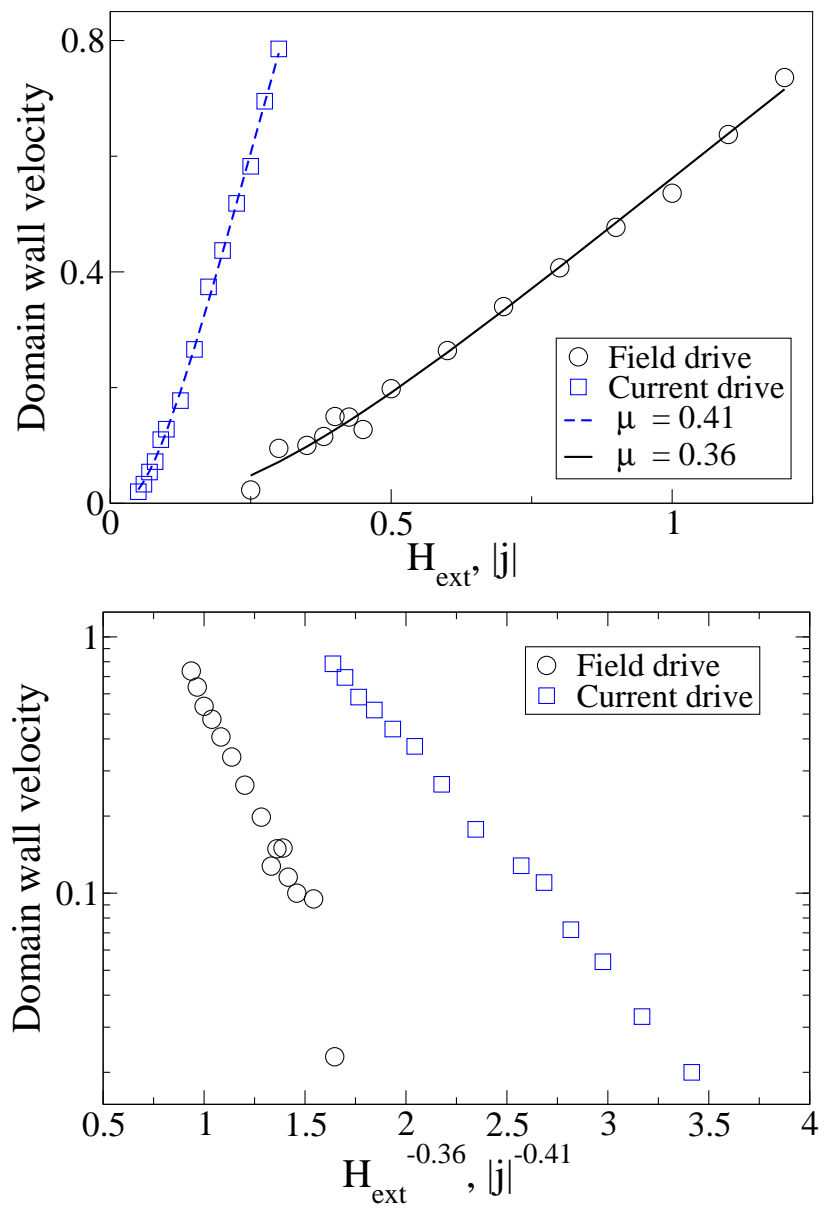

Fig. 4. The average creep velocity versus the external field $H_{e x t}$ and current J. The top panel shows the data (symbols) along with non-linear fits of the form of Eq. (3) (solid and dashed lines). The lower panel shows the same data scaled according to the fitted values of the creep exponent $\mu$.

a different system with a different wall configuration where different creep exponents were found in the two cases [15]. Thus, a detailed experimental study of the creep dynamics of transverse domain walls in nanostrips would be interesting, to see if the simplified model presented here captures the statistical properties of the true dynamics. Notice also that the non-linear behaviour of the domain wall velocity versus the applied external drive is out of scope of any point particle based models, where one would expect $v(f) \sim f$.

Stochastic velocity fluctuations of domain wall motion in nanostrips have been observed also in experiments [16], [17], [18]. This might suggest that these experiments are typically probing the creep regime, with the velocity distribution arising from the random sequence of pinning and depinning events governed by thermal fluctuations. From the point of view of practical spintronics-based applications, this presents a fundamental problem: how to displace a domain wall within a nanostrip in a controllable fashion? From the point of view of low power consumptions of the devices, it might be desireable to use low driving forces, but we have seen that within this regime the relative fluctuations in the domain wall dynamics are large. Using stronger external drives leads to different problems, as the wall magnetization starts to precess around the long axis of strip, something that in the presence of disorder could also happen in a stochastic manner. As the translational and phase degrees of freedom of the wall are coupled, this would presumably affect also the translational motion of the domain wall.

\section{ACKNOWLEDGMENT}

LL wishes to thank Academy of Finland for financial support.

\section{REFERENCES}

[1] G. S. D. Beach, M. Tsoi, and J. L. Erskine, J. Magn. Magn. Mater. 320 $1272(2008)$.

[2] S. S. P. Parkin, M. Hayashi, and L. Thomas, Science 320, 190 (2008).

[3] G. S. .D. Beach et al., Nature Mater. 4, 741 (2005); R. Cowburn and D. Petit, Nature Mat. 4, 721 (2005).

[4] D. A. Allwood et al., Science 309, 1688 (2005).

[5] E. Martinez et al., Phys. Rev. B 75, 174409 (2007).

[6] Y. Nakatani, A. Thiaville, and J. Miltat, Nature Mat. 2521 (2003).

[7] M. T. Bryan, D. Atkinson and R. P. Cowburn, J. Phys: Conf. Ser. 17, 40 (2005).

[8] E. Martinez et al., Phys. Rev. Lett. 98, 267202 (2007)

[9] R. D. McMichael et al., IEEE Trans. Magn. 334167 (1997).

[10] Y. Nakatani, A. Thiaville, J. Magn. Magn. Mater. 290-291, 750 (2005).

[11] M. Laufenberg et al., Appl. Phys. Lett. 88, 052507 (2006).

[12] S. Zhang and Z. Li, Phys. Rev. Lett. 93, 127204 (2004).

[13] M. J. Donahue and D. G. Porter, OOMMF User's Guide, Version 1.0, Interagency Report NISTIR 6376, National Institute of Standards and Technology, Gaithersburg, MD (Sept 1999).

[14] http://www.susqu.edu/brakke/evolver/evolver.html

[15] M. Yamanouchi et al., Science 317, 1726 (2007).

[16] G. Meier et al., Phys. Rev. Lett. 98, 187202 (2007).

[17] P. Möhrke et al., J. Phys. D 41, 164009 (2008).

[18] A. Vanhaverbeke, A. Bischof, and R. Allenspach, Phys. Rev. Lett. 101, 107202 (2008) 